\title{
Evaluation of the Effectiveness of a Web-Based e-Learning System for Tertiary Educational Institution
}

\author{
Touhid Bhuiyan, Sharmin Khan, and Aynun Nahar
}

\begin{abstract}
Thegrowing demand of e-learning system in academia is well accepted worldwide. One of the vital missions of e-learning is to allow people to learn for personal accomplishment or to earn a professional degree, without physically attending a traditional university or academic setting. E-learning can be applied for all levels of schooling from grade school to graduate degrees, and is versatile enough to accommodate all learning styles. A good number of researchers all over the world have already worked for developing different types of web based e-learning system. In this paper, we have presented our proposedweb-based e-learning system which is essential for any tertiary educational institution. Both students and teachers are tied up in this system. Overall it's a guided learning for student and a great way to change our typical learning system but not replacement of teaching, it's only the supplement idea for teaching which helps to learn easily and improve the productivity. We have also evaluated the effectiveness of our proposed system by conducting a survey, analyzed and presented the result.
\end{abstract}

Index Terms-E-learning, computer based training (CBTs), data flow diagram (DFD), survey, evaluation, web based training (WBTs).

\section{INTRODUCTION}

E-learning can occur in or out of the classroom. It can be self-paced, asynchronous learning or may be instructor-led, synchronous learning.It is suited to distance learning and flexible learning, but it can also be used in conjunction with face-to-face teaching, in which case the term blended learning is commonly used.Itis defined as electronically mediated asynchronous and synchronous communication for the purpose of creating and performing knowledge [1]. The technological foundation of e-learning is internet and associated communication technology like cell phone, iPod etc. E-learning is the use of technology to allow people to learn anytime from anywhere. E-learning can consists of training, the delivery of just-in-time information and guidance from experts.Actually E-learning is a system where a person has some information which need for him to communicates effectively, collaborate and train. All these things put together constitute e-learning. There is no worldwide definition of e-learning. E-learning refers to any form of learning that we are familiar with or which we can access through Web-enabled technology [2].

Manuscript received May 12, 2013; revised August 20, 2013.

Touhid Bhuiyan was with the Department of Computer Science and Engineering, East West University and now is with the Department of Software Engineering, Daffodil International University, Bangladesh (e-mail: t.bhuiyan@daffodilvarsity.edu.bd).

Sharmin Khan and AynunNahar are with the Department of Computer Science and Engineering, East West University, Bangladesh.
The term "e-learning" was first used in October of 1999. The expression specifically referred to learning using the Internet or other interactive or electronic media sources. It was also termed as "online learning," according to the e-Learning fundamentals website. E-learning is a type of distance learning because the student has the freedom to learn lessons and complete assignments outside the classroom.

Presently, it is generally thought that new technologies can firmly help in education. In young ages especially, children can use the vast interactivity of new media, and expand their skills, knowledge, and perception of the world, under their parents monitoring [3]. Though traditional education cannot be replaced, but in this era of fast technological advance and minimization of distance through the use of the Internet, everyone must be equipped with basic knowledge in technology, as well as use it as a medium to reach a particular goal.

There are different ways to present eLearning. Mainly they are divided by two types. They are synchronous and asynchronous. Synchronous means "in the intervening time," involves interaction of participants with an instructor by the Web in real time. For example - VCRs or Virtual class rooms that are nothing else but real classrooms online. Again asynchronous means "not in the intervening time," permits the participants to complete the WBT (Web-based training) at their own pace, without live communication with the instructor. Mainly, it is information that is available on a self-help basis, 24/7. The advantage is that this kind of e-learning offers the learners the information they need whenever they need it. It also has relations amongst participants through message boards, bulletin boards and discussion forums. These contains computer based training(CBTs) elements on CD-ROM's, Web based training accessed through internet (WBTs) or through well written articles and other write ups. From the Fig. 1 we can get complete view of e-learning.

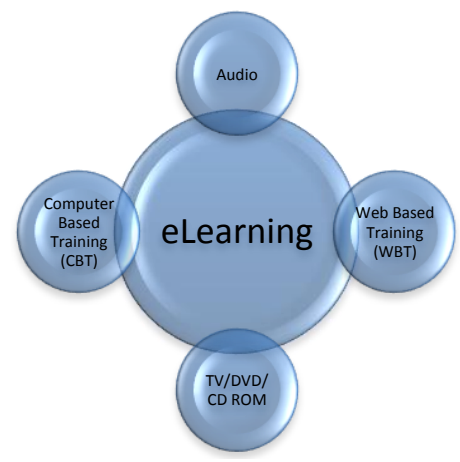

Fig. 1. Different types of e-learning.

Researcher all over the world already developed many e-learning programs and software but most of them are 
expensive and not flexible. In this paper we develop a web based e-learning system for teaching institution. Overall our proposed system is a guided learning for student and a great way to change our typical learning system but not replacement of teaching. It's only the supplement idea for teaching which helps to learn easily and fill up their lickings. The most important thing of our system is, that it is cheaper and user friendly.

The organization of our paper is as follow: Section II describes the methodology which include data flow diagram, ER diagram and database design of our proposed system, Section III describes about testing and implementation of our system, Section IV describes the evaluation of our system by presenting the survey result and analysis of findings, And finally Section V draws conclusions with some remarks on future works.

\section{Methodology}

In this section we will give an introduction of our system and also give a comprehensible idea regarding implementation our system. In this system we use some programming languages and database tools like MySQL, AJAX, CSS, XML, IP, PHP and HTML.

\section{A. Data Flow Diagram}

Data flow diagram is a graphical representation of the flow of data through an information system [4].The data flow diagram is a model describing the information exchange processes in the system. The DFD model is composed of the following main object types: processes, internal objects, datarepositories, and data flows. Internal objects feature the modeled process environment; data repositories store dataneeded by the processes. The processes transform their input data flows into the output data flows which may be sent from one system element to another [5]. Our proposed system design consisted in several stages:

TABLE I: STAGES OF OUR SYSTEM

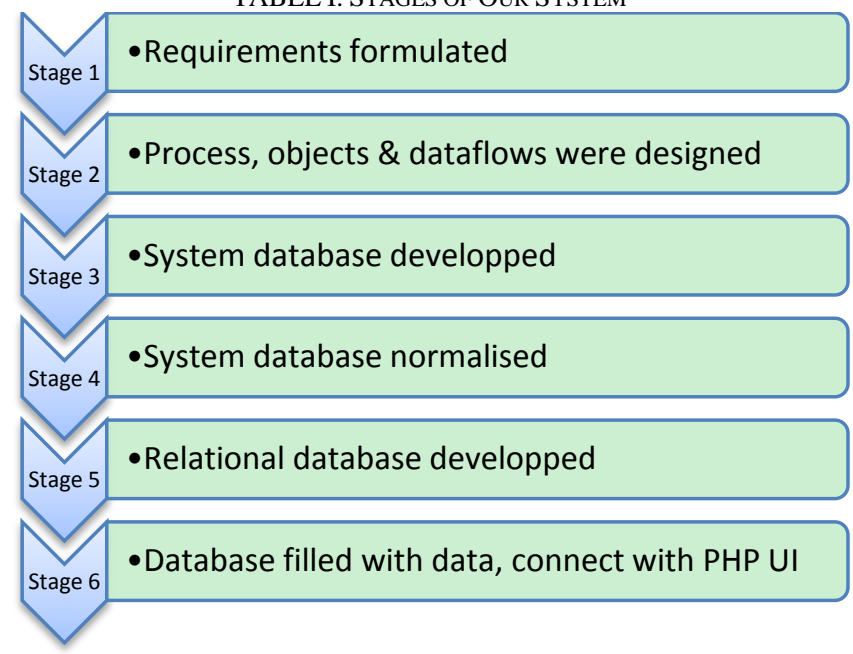

An E-R diagram is the Entity Relationship Diagram, showing the relationship between different entities in a process. The E-R diagram developed during the system design process is shown in Fig. 2. Processes are depicted in the diagram as rectangles with the rounded corners.

For databases, the E-R diagram [4], [6]-[8] refers the entity relationship diagram used to represent in the graphical way dependencies between entities in the database, other than the DFD diagram, focused on modeling the information interchange within the system [9], [10]. It was enough to use the DFD model developed earlier to determine the properties - attributes - of entities, as they were initially formulated in data flows definitions.

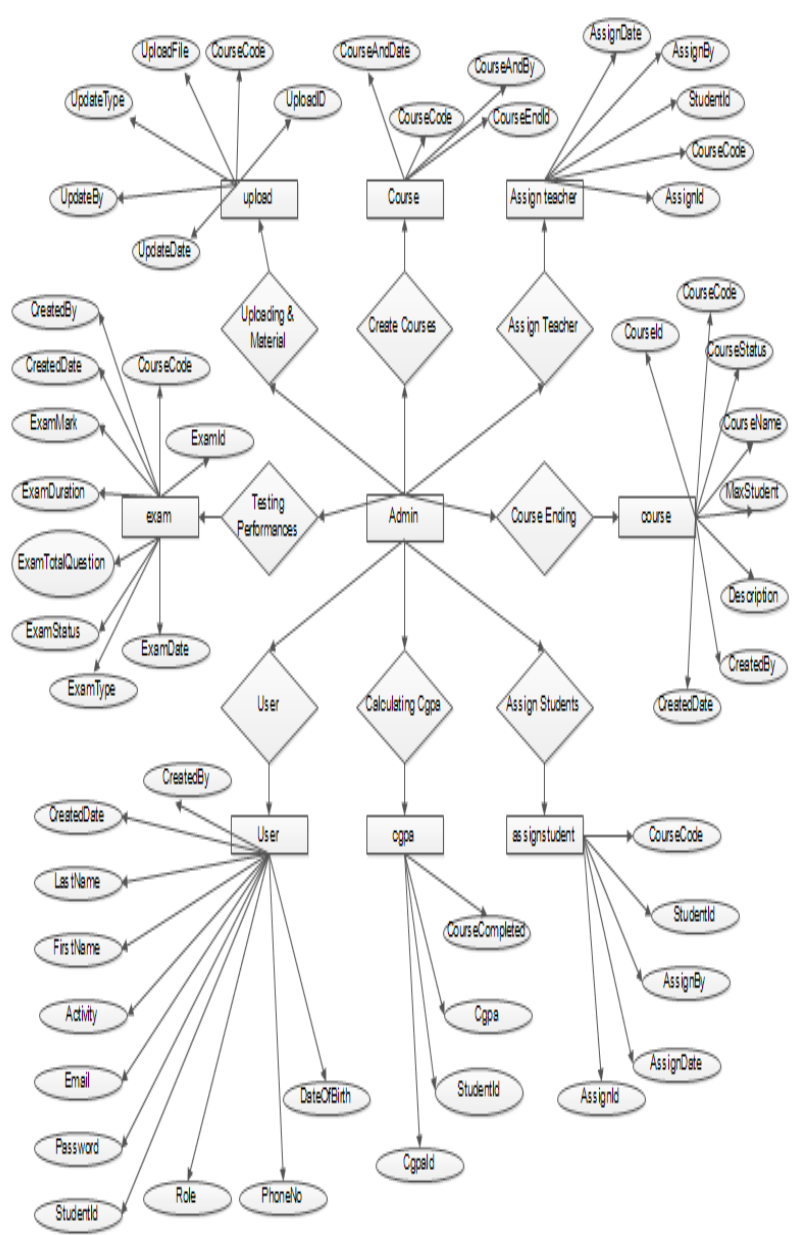

Fig. 2. E-R diagram of our proposed system.

\section{B. Database Design}

Database design is the method of constructing an exhaustive data model of a database. Almost all web applications are motivated by a database, and with Core Data, Cocoa applications are starting to have true database back ends. The proposed system is developed by PHP plus MySQL, where PHP is the core programming language and MySQL is the web database[11]. Eighteen MySQL data tables are designed for our proposed system which shown, in Fig. 3. In this system admin has all the power to manage and update the system. Teachers can monitoring the system and can give essential materials and student's acts as learner as well as regular user of the system.

\section{System TESTING AND IMPLEMENTATION}

The philosophy behind testing is to fine errors. The common view of testing is that it is performed to prove that there are no errors in a program. However it is virtually impossible to prove that no program will be free and clear of errors. Therefore the most useful approach and practical 
approach is with the understanding that testing is the process of executing a program with explicit intention of finding errors that is, making the program fail. Executing a program in a simulated environment performs verification. It is sometimes called Alpha Testing. Validation is the process of using the software in a live environment in order to find errors. It can be called as Beta Testing. System testing is the stage of implementation, which aims at ensuring that the system works accurately and efficiently before actual operation commences. No program or system design is perfect; communication between the user and the designer is not always complete or clear, and time is usually short. The result is errors and more errors. The number and nature of errors in a design depend on several factors. These are Communication between the user and the designer, the programmer's ability to generate a code that reflects exactly, the system specificationand the time frame for the design [12], [13].

\section{System Evaluation}

To evaluate the effectiveness of our proposed learning system, we have conducted an online survey. The details of the survey are described briefly in this section.

\section{A. Study Objective}

The major objective of this survey is to collect information about the user's view regarding the impact of a LMS in our education system. In this survey the questioner to obtain information about two main sub-topics listed below:

- Acceptance and impact of using LMS in education sectors

- The basic intended features of users of Bangladesh in a LMS

\section{B. Study Design}

An online survey methodology was chosen in order to maximize the speed of data collection and anonymity of participants. The survey was designed by using SurveyMonkeys.com and it contained 20 questions. The questions were developed based on key issues in the academic and lay literatures and experimental knowledge. Questionnaire is a standard and structured instrument of a survey [14]. Often they are the only feasible way to reach a number of respondents large enough to allow statistically analysis of the result. While designing the questionnaire, it should be focused on appropriate closed ended questions and administered in a standard way. When fairly straightforward information is required and focused on "what" occurs rather than "why" or "how", questionnaire has its best use in such a situation. The main objective of this survey was to find the impact of a learning management system on our education system. For this reason, survey questionnaire has designed with clear, simple and precise closed ended questions to obtain the straightforward information with predetermined responses about the users' opinion. One has to aware that sometimes it is difficult to capture the richness of meaning in survey questions. That's why the design of the questionnaire must be self-explanatory statements.An introductory statement has been given with the study title, its organization and aims of the survey. Consistent and clear instructions have also been given throughout the questionnaire. The general steps to design and administer a questionnaire:

- Defining the objective of the survey

- Determining the sampling group

- Writing the questionnaire

- Administering the questionnaire and

- Interpretation of the results.

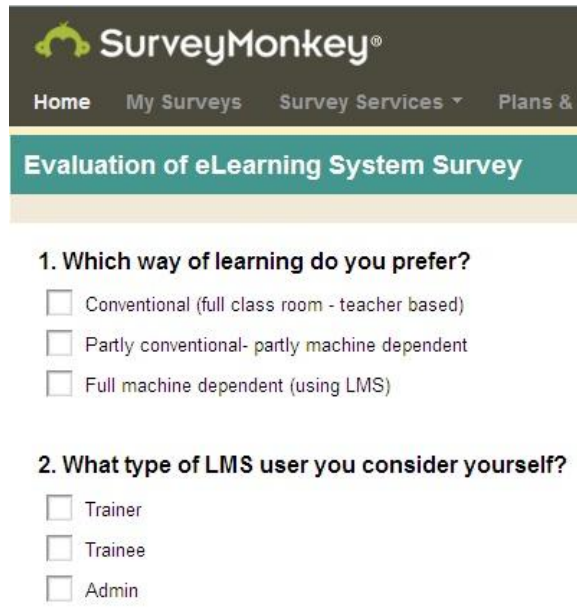

Fig. 3. A screen shot of used online survey tool.

In creating a survey, the investigator only should ask what is necessary and what might be interesting. Trying to answer too many things usually means none of them are answered well. For this reason, the questions were kept to a minimum number. It also stated that "You will remain anonymous and any identifiable information you provide will changed. Information you provide will be held on SurveyMonkey's server, however, SurveyMonkey guarantees that the data will be kept private and confidential". The survey was piloted and refined before going live.

\section{Requirement and Data Collection}

Respondents were recruited using strategic opportunistic sampling. Emails were sent to different University students and teachers. The survey link for this study was also published through the social network Facebook. Data were collected between January, 2013 and April, 2013. Due to the lack of available time; the survey was limited to specific people only. The time limitation of this survey also limits the number of respondents.

\section{Respondents}

A total of 967 respondents, from different universities of Bangladesh participated in the study collected online. Though there was not specific user for the survey, the invitation email to participate in the online survey was sent to only university students and teachers. Respondents included both male and female online users of different age groups.

\section{E. Results}

Respondents included students who have used online educational tools and family members of such students as well as teachers and university faculty who had substantial experience and expertise with such online tools. Each of these respondents willingly shared their stories and, in doing so, helped inform the findings included in this paper.

This developed software was only very common features of a LMS, other popular features like email, chat, online resources, link, and messenger service and so on are not 
available in the software. There were total 967 persons as the respondents of this survey through SurveyMonkey.com within the allocated time period. The participants had to answer all the questions [15]-[18].

\section{Acceptance and impact of using LMS in education sectors}

At first we wanted to know trainers view on the way of learning using the question-"Which way of learning do you prefer?" Among the respondents 10\% likes conventional (full class room - teacher based), $60 \%$ likes partly conventional-partly machine dependent and the rest 30\% likes full machine dependent (like Learning Management System).

Before going further through the survey user must have knowledge about learning management system. The ratio of having knowledge about LMS is $50 \%-50 \%$.About $9.09 \%$ users was trainer, $72.73 \%$ users was trainee and $18.18 \%$ among users was admin type user of this survey respondents.Almost $63.64 \%$ users were working and $36.36 \%$ users were non-working.More than $45 \%$ users uses Moodle, $36.36 \%$ users use BlackBoard, less than $10 \%$ users uses Canvas LMS.

Little more than $80 \%$ users have had a positive overall experience with learning management system.

\section{The basic intended features of users of Bangladesh in a} LMS

Most of the user among survey respondents response that used LMS must be so easy to use (see Fig. 4). According to that proposed LMS (learning management system) is very easy to use to $18.18 \%$ users, reasonably easy to $81.82 \%$ users.

$27.27 \%$ users strongly agreed that proposed LMS increase the interactivity and improve learning and rest $72.73 \%$ users agreed that proposed LMS increase the interactivity and improve learning. $72.73 \%$ users think that "User activity tracking" is more specific and useful inourproposed system.

"Listening to or viewing instructional media" was one of the useful features of proposed LMS. More than $45 \%$ users think it is very useful and $18,18 \%$ users did not use the features. According to response, more than $75 \%$ users proposed LMS is more flexible than others.

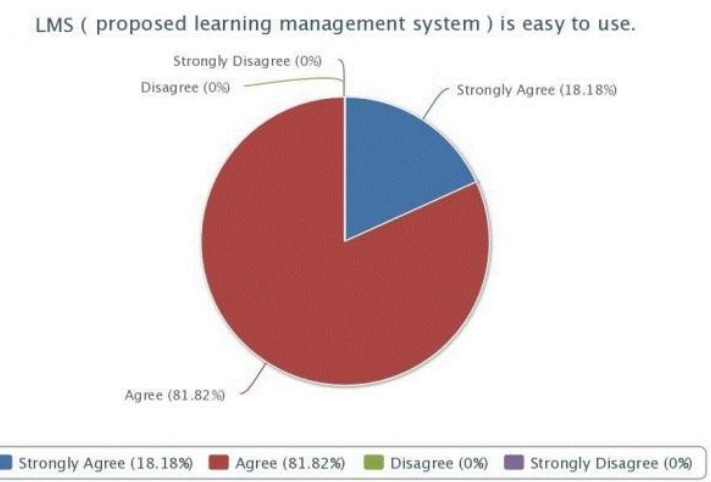

Fig. 4. Easy to use.

About $80 \%$ users customized proposed LMS to their choice, less than $20 \%$ did not customize it. Almost all the users consider proposed LMS is less expensive comparatively. And more than $80 \%$ user can easily control the full system of proposed LMS according to their choice.

Almost all users suggest having a LMS in Bengali language; they think it will be useful for our country. Most users want to use a LMS in their Android device; according to them it will be more popular in young generation of our country. Near about $90 \%$ users think LMS of lower cost like our proposed system will be more popular \& effective in universities in our country. Over $40 \%$ users find proposed LMS more user friendly than other users (see Fig. 5).

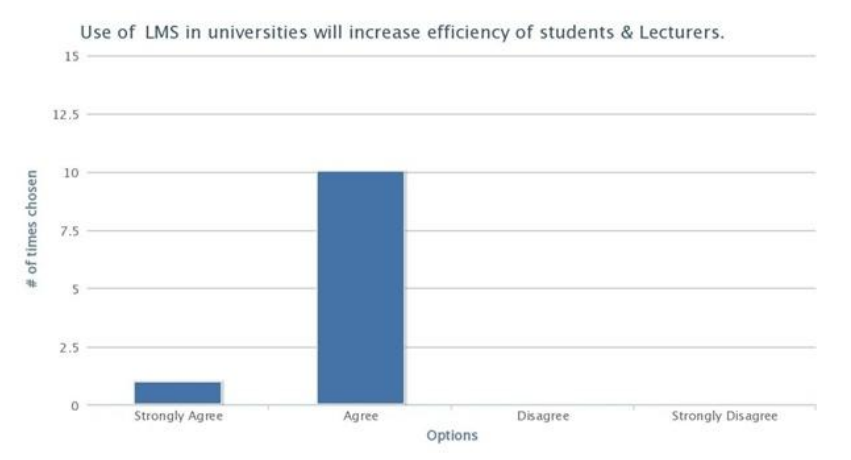

Fig. 5. Efficiency improvement.

Most respondents think that using of proposed LMS in universities will increase efficiency of students \& Lecturers. Comparing both conventional and LMS based learning and after the analysis I found $45 \%$ learners have positive change in performance \& efficiency of a student after using a LMS, $40 \%$ learners have no change in performance \& efficiency of a student after using a LMS, $15 \%$ learners have negative change in performance $\&$ efficiency of a student after using a LMS (see Fig. 6).

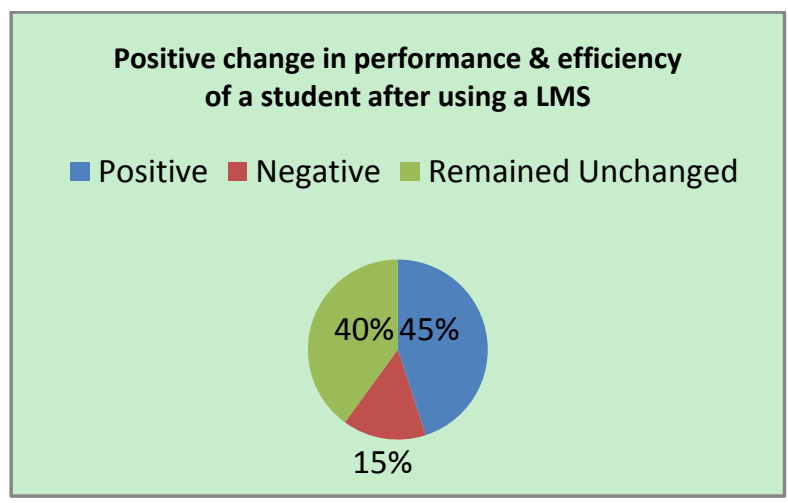

Fig. 6. Performance improvement

\section{F. Findings and Discussion}

Overall results demonstrated that more than one third of respondents who use assistive technology to access online educational tools reported the experience as a successful use/access. Open ended questions gave respondents the opportunity to share their personal stories. Data indicated that several of the most important features of online educational tools posed the most problems for those who used screen reading or screen magnification software. In nearly every instance, respondents indicated features that were inaccessible.

The ability of respondents to effectively use access technology was considered in this report. As mentioned, respondents included students who have used online educational tools, family members of such students, as well as teachers and university faculty who had substantial experience and expertise with such online tools. Consistent trends among participant responses were substantial enough 
to negate this otherwise potentially salient factor.

Even though the survey did not ask for suggestions about how to best accommodate for many of the most problematic features that people may want with online education, respondents provided suggestions. Those involved with online education are encouraged to adhere to the aforementioned suggestions and those who have the potential to develop the technical expertise to better the situation are urged to resolve many of the problematic features. It is noted that adhering to these suggestions can give students a better chance for successful access with the usable features of online education until the problematic features are remedied by the necessary experts. It is also noted that adhering to these suggestions can give all students a notable better chance for successful online education experiences.

Efforts to remedy the situation should be grounded in bettering the problematic features that prevent full and equal access for people. Respondents shared their personal stories through the open ended questions included in this survey. Parents must access online education materials for their high school children. University students are unable to complete posts-secondary degrees that involve the use of online educational tools due to accessibility barriers that are too substantial to overcome. These are unacceptable circumstances in this time of technological prominence when computers have the capacity to bridge the digital divide.

\section{CONCLUSION AND FUtURE WORKS}

Evaluating a system for e-learning is challenging. In this paper we proposea web based e-learning system which can be easily implemented in the teaching institution of developing countries because of its low cost and effort. We have also conducted a survey, analyzed the result and presented in this paper. Most of the teaching institutions of developed countries are now using information and communication technology to automate their education process. If we the developing countries want to keep pace of the progress with the rest of the world, we must have to do the same. Projects like this will help to automate an important sector of our education. Through our proposed e-learning system anyone can visualize the usefulness and efficiency in the education. The survey results also indicate that the system is easy to use and has positive effects of its principal objective.

In future we have planned to make our system more effective and user friendly. We also want to add some additional facilities to our system like SMS alert system, text message notification over mobile, Facebook application, educational game development, Cloud System and make our system for android system.

\section{REFERENCES}

[1] D. R. Garrison, E-Learning in the 21st Century, United Kingdom: Taylor \& Francis, pp. 1-15, March 2, 2011.

[2] M. Suemitsu, Y. Akimitsu, T. Kiyan, Y. Ohshima, K. Kozono, S. Kamaga, and H. Akiyama, "Development of KUMA system for rapid e-Learning," in Proc. 9th International Conference on Information Technology Based Higher Education and Training, 2010, pp. 136-139.

[3] Q. Yang, J. Sun, and J. Wang, "Semantic web-based personalized recommendation system of courses knowledge research," in Proc. International Conference on Intelligent Computing and Cognitive Informatics, 2010, pp. 214-217.
[4] R. A. Elmasri and S. Navathe, Fundamentals of Database Systems, 6th ed., Addison-Wesley, 2010, ch. 4, pp. 51-53.

[5] M. Gumiska, "Employment of the internet technologies in promoting the distance learning offers, based on the Internet Teaching Centre for Students example," MSc diploma Project, Gliwice, 2006.

[6] M. Kang, Y. Park, J. Kim, E. Yoo, and S. Cho, "Investigating a structural relationship among different variables in using web-based inquiry learning for elementary science classes," in Proc. World Conference on Educational Multimedia, Hypermedia and Telecommunications, 2012, pp. 1761-1772.

[7] L. Welling and L. Thomson, PHP and MySQL Web Development, 4th ed., Addison-Wesley Professional, 2008

[8] F. Abel, I. I. Bittencourt, E. Costa, N. Henze, D. Krause, and J. Vassileva, "Recommendations in online discussion forums for e-learning systems," IEEE Transaction Learning Technologies, vol. 3, pp. 165-176, April-June 2010.

[9] L. Ullman, PHP and MySQL for Dynamic Web Sites, Peachpit Press, 2003.

[10] J. C. Meloni, PHP, MySQL i Apache for Everyone, Helion, Gliwice, 2005.

[11] W. Choi, A. Kent, L. Lea, G. Prasand, and C. Ullman, PHP4 Starter, Helion, Gliwice, 2002, (in Polish).

[12] L. Titchkosky, M. Arlitt, and C.Williamson, "A performance comparison of dynamic web technologies," in Proc. 11th IEEE/ACM International Symposium on Modeling, Analysis and Simulation of Computer Telecommunications Systems, 2003.

[13] J. Losec, Z. Xiaobin, and W. Jingli. "Affective bracket: students' emotional and academic intersection of teachers' knowledge," Open Education Research, vol. 5, 2009.

[14] J. Lennon and H. Maurer, "Why it is difficult to introduce e-learning into schools and some new solutions," Journal of Universal Computer Science, vol. 9, no. 10, 2003, pp. 1244-1257.

[15] E-learning Platform of Institute of Engineering Materialsand Biomaterials. (June 13, 2013). [Online]. Available: http://www.platforma.imiib.polsl.pl

[16] W. Willard, Web Page Design. Fundamentals, Wydawnictwo Edition 2000, Kraków, 2001 (in Polish).

[17] Getting Educational Systems Talking Across Leading-Edge Technologie. (June 13, 2013). Work Package 3, D0301 Design and Specification of the Resource Discovery Service, 1999. [Online]. Available: http://www.fdgroup.co.uk/gestalt/

[18] V.-A. Romero-Zaldivar, A. Pardo, D. Burgos, and C. D. Kloos, "Monitoring student progress using virtual appliances: A case study," Computers \& Education, vol. 58, no. 4, pp. 1058-1067, 2012.

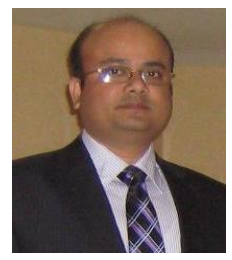

Touhid Bhuiyan is working as an associate professor at the Department of Software Engineering, Daffodil International University (DIU). His research interests are in intelligent recommendations, social network, trust management, database management and e-Learning in general. Before joining at DIU, he was employed by several renowned organizations including the Queensland University of Technology, University of Western Australia, University of Western Sydney and Central College Sydney. He has more than 16 years experience in teaching, research and working at the IT industry in Australia, Singapore and Bangladesh Previously he also served the East West University and the People's University of Bangladesh.

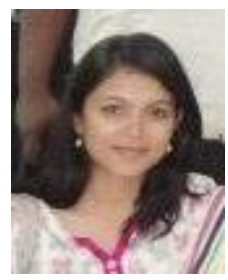

Sharmin Khan is the student of the Department of Computer Science and Engineering, East West University. Her research interest includescoding optimization, data mining, implementation of automated system, artificial intelligence etc. She is the member of the work group "Web-based e-Learning Implementation Project". She is planning to do her research-masters in the area of e-Learning.

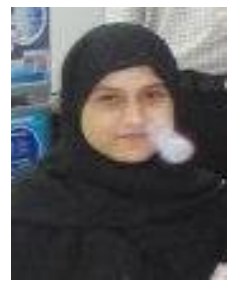

Aynun Naharis the student of the Department of Computer Science and Engineering, East West University. Her research interest includes eLearning, technology-based education, data analysis, web databasemanagement, implementation of automated system etc. She is the member of the work group "Web-based e-Learning Implementation Project".Currently, she is conducting a survey on the impact of the e-Learning tools used in the non-academic setting. 